\title{
A Case of Otogenic Brain Abscess Causing Loss of Consciousness
}

\author{
Min Kyu Kwak, Jae Ho Chung, Seung Hwan Lee, and Chul Won Park \\ Department of Otolaryngology-Head and Neck Surgery, College of Medicine, Hanyang University, Seoul, Korea
}

\author{
Received October 30, 2013 \\ Revised April 2, 2014 \\ Accepted May 15, 2014
}

\author{
Address for correspondence \\ Jae Ho Chung, MD \\ Department of Otolaryngology- \\ Head and Neck Surgery, \\ College of Medicine, \\ Hanyang University, \\ 222 Wangsimni-ro, Seongdong-gu, \\ Seoul 133-791, Korea \\ Tel +82-31-560-2360 \\ Fax +82-31-566-4884 \\ E-mail jaeho.chung.md@gmail.com
}

Acute or chronic otitis media can cause intracranial complications, one of the most serious being brain abscess. Empirical antibiotic treatment and proper surgical management should be considered to avoid fatal consequences. However, proper extent and optimal timing of surgical intervention are still matters of debate. We present a case of a 31-year-old man who presented with acutely altered mental status, caused by otogenic brain abscess who we treated successfully with antibiotics and otologic surgery and no neurosurgical treatment.

Korean J Audiol 2014;18(2):76-79

KEY WORDS: Otitis media · Brain abscess · Complication.

\section{Introduction}

Acute and chronic otitis media can cause intratemporal and intracranial complications. Intracranial complications include meningitis, brain abscess, epidural abscess, subdural empyema, and lateral sinus thrombosis. These conditions are potentially dangerous or even fatal. With the development of new antibiotics and improvements of inpatients care, the incidence of intracranial complications has been remarkably reduced nowadays compared with the pre-antibiotic era. However, mortality of these complications is still high and improper use of antibiotics can change or conceal the characteristics of the disease which can make diagnosis difficult. Otogenic brain abscess is the second most common intracranial complication of acute otitis media ( 0.5 percent $){ }^{1)}$ In the past, the mortality rate of otogenic brain abscess was 14 to 35 percent, but has now decreased to 3 percent. ${ }^{2)}$ The selection of antibiotics and appropriate surgical intervention are mighty important in the treatment of otogenic brain abscess. However, the extent of surgical procedures and optimal timing for these intervention

This is an Open Access article distributed under the terms of the Creative Commons Attribution Non-Commercial License (http://creativecommons. org/licenses/by-nc/3.0/) which permits unrestricted non-commercial use, distribution, and reproduction in any medium, provided the original work is properly cited. are still matter of debate. ${ }^{2)}$

We report a patient of otogenic brain abscess causing loss of consciousness who was treated with systemic antibiotics and proper surgical management.

\section{Case Report}

A 31-year-old male presented at the emergency department with acutely altered mental status. He had suffered fever, headache, otalgia, and otorrhea for the previous three weeks. He had been diagnosed with acute otitis media at the private clinic and had taken antibiotics. At initial presentation, his body temperature was $39.5^{\circ} \mathrm{C}$, pulse rate $99 /$ minute, respiratory rate 28/minute, and blood pressure 160/100 $\mathrm{mm} \mathrm{Hg}$.

On physical examination, his right external auditory canal was swollen and purulent discharge was expelled from a small perforation of the tympanic membrane which was bulging and hyperemic. Culture and drug sensitivity tests of the discharge were performed to determine the causative organism and appropriate antibiotics. Swelling of postauricular area was not identified. The patient appeared in a state of stupor. A blood test showed white blood cell (WBC) $28900 / \mathrm{mm}^{3}$ and C-reactive protein $13.6 \mathrm{mg} / \mathrm{dL}$. Cerebrospinal fluid (CSF) study was performed to rule out meningitis and intracranial infections. 
In lumbar puncture, there was increased pressure of CSF which was turbid with elevated WBC count $\left(12800 / \mathrm{mm}^{3}\right)$. Computed tomography (CT) scans of the brain and the temporal bone were performed to find the cause of altered mental state. Temporal bone CT scan revealed that the right tympanic cavity and mastoid antrurm were filled with soft tissue density. And brain CT scan showed an enlarged right lateral ventricle which had been identified on the previous CT scan regular checking up (Fig. 1).

The patient was admitted to neurology department with the initial diagnosis of bacterial menigoencephalitis and treated with empirical antibiotics (3rd generation cephalosporin and vancomycin) in the intensive care unit (ICU). Mechanical ven-

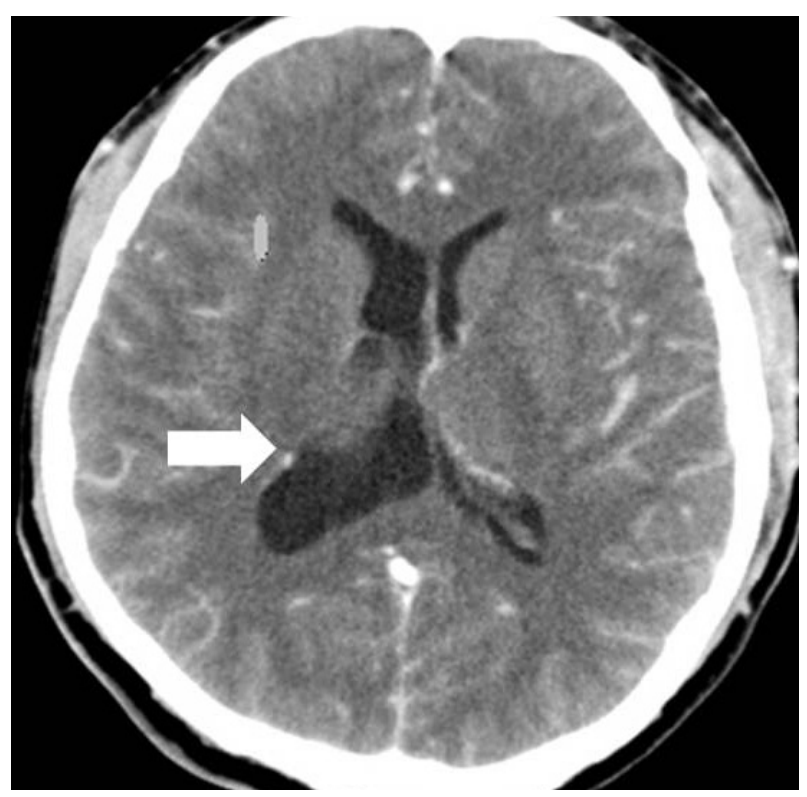

Fig. 1. An axial scan of brain computed tomography with contrast enhancement demonstrates asymmetric dilated right lateral ventricle (white arrow). tilation care was started to support decreased respiration effort. And steroids and diuretics were administered to reduce intracranial pressure.

On the third day in the ICU, a consultation for otolaryngology department was requested because of unchanged mental state and findings of otitis media on temporal bone CT. The patient underwent myringotomy and ventilation tube insertion. During the operation, serious discharge was expelled from the myringotomy site. At that time, Streptococcus pneumonia that was susceptible to ceftriaxone $(\leq 0.06)$ was isolated from discharge from the ear and CSF.

As the patient's general conditions and mental status were getting better, he was transferred to the general ward. In spite of a full recovery in mental status, he complained a severe headache and was not responding to pain killers. On his thirteenth day in the hospital, another radiologic test was performed to evaluate the cause of intractable headache. About $1.6 \times$ $1.2 \times 1.0 \mathrm{~cm}$ sized encapsulated abscess was noted at the right thalamic area on the brain magnetic resonance imaging (MRI)(Fig. 2A). On the temporal bone CT scan, the middle ear cavity and all of the mastoid air cells were still filled with soft tissue (Fig. 3)

Guided by the results of pure tone audiometry, conductive type hearing loss of right ear (air conduction; $30 \mathrm{~dB}$, bone conduction; $18 \mathrm{~dB}$ ) was checked. On the twenty seventh day of hospitalization, a second brain MRI was performed to determine the extent of abscess. The size of the brain abscess was decreased slightly on this MRI scan (Fig. 2B).

On the thirty second day of disease, otologic surgery was planned for prolonged intermittent fever and no improvement of otitis media as seen on the temporal bone CT. Upon simple mastoidectomy, the mastoid cavity and middle ear were filled with reddish granulation tissue (Fig. 4), while the ossicles and
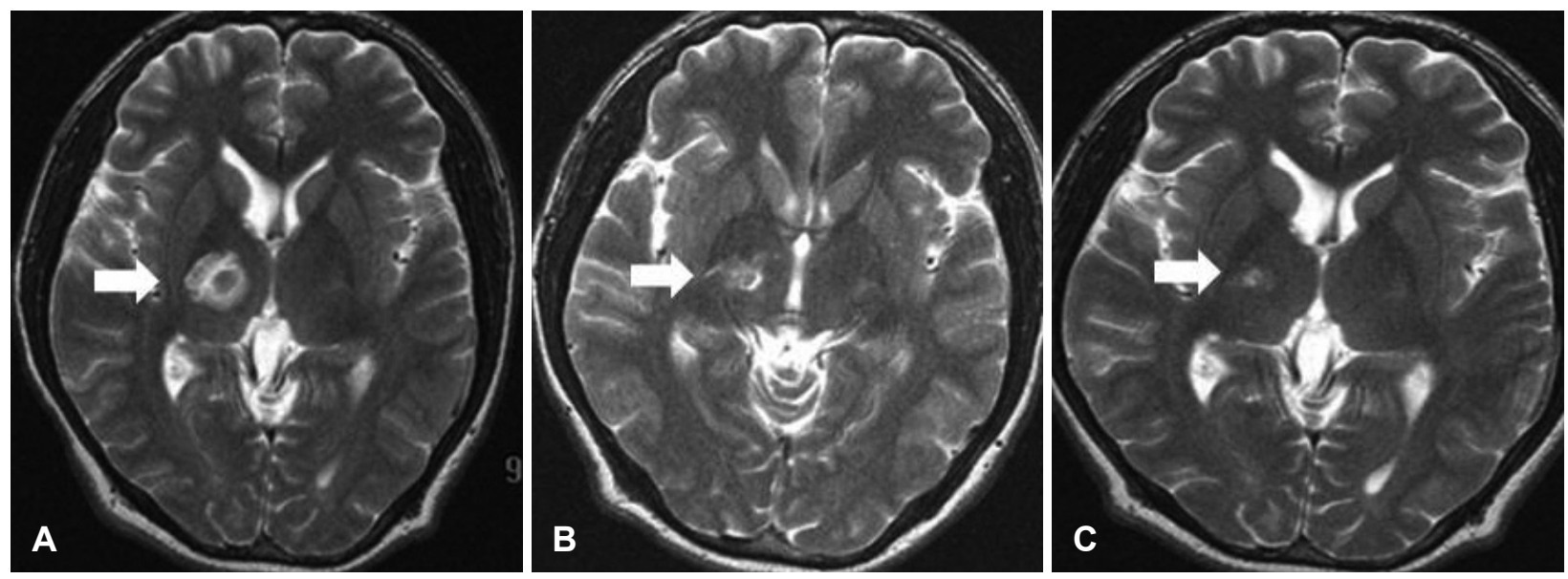

Fig. 2. T2-weighted magnetic resonance images with contrast enhancement on the 13th (A), 27th (B), and 39th (C) days of hospitalization. With the antibiotics treatment and simple mastoidectomy, the rim-enhancing brain abscess in the lateral inferior part of the right thalamus became smaller with time (white arrows). 


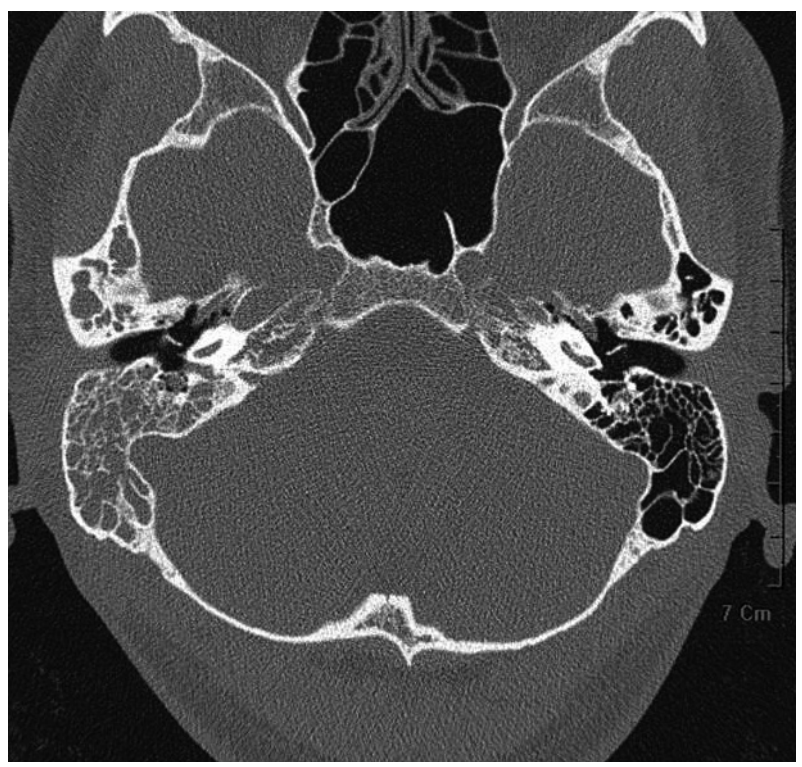

Fig. 3. Temporal bone computed tomography image on the 17th day of hospitalization. All air cells in right temporal bone were filled with soft tissue. Aeration of the tympanic cavity was fair by virtue of a ventilation tube.

the facial canal were intact. Localized destruction of attic and tegmen were accompanied with partial exposure of the dura mater that covers the floor of the middle cranial fossa. Tegmen defect might be a direct propagation route of inflammation causing brain abscess. However, considering the location of the abscess, the possibility of hematogenous propagation could not be excluded.

After mastoidectomy, antibiotic therapy was maintained. On postoperative day seven, the right thalamic brain abscess had nearly disappeared on brain MRI (Fig. 2C). The patient was discharged without neurologic sequelae at ten days after the surgery.

\section{Discussion}

Otogenic brain abscess is the accumulation of pus in the cerebrum or cerebellum developing with encephalitis, caused by pyogenic microorganisms originating from inflammatory processes in the middle ear cavity. Acute and chronic otitis media equally cause otogenic brain abscess. ${ }^{3,4)}$

Possible propagation pathways of brain abscess consist of preformed pathway, direct extension, as well as hematogenous routes. Among these, the most common cause is direct extension of infection through osteitic bone. ${ }^{5)}$ Otogenic brain abscess usually presented as a solitary abscess rather than multiple forms. It develops in the temporal lobe two to four times as often as in the cerebellum. But there are many different reports about location of abscess. ${ }^{5,6)}$ Upon the development of antibiotics, the incidence of otogenic brain abscess decreased

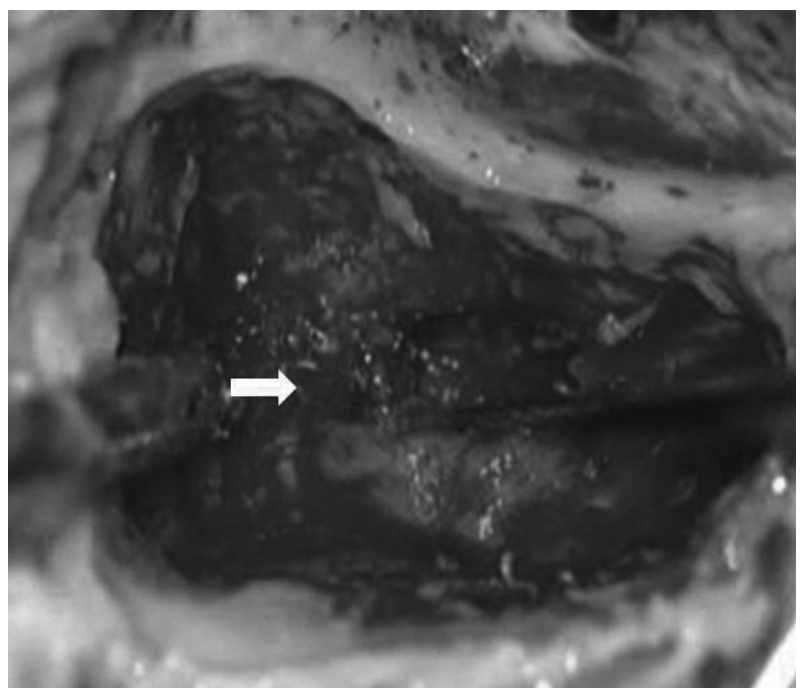

Fig. 4. Operative findings of simple mastoidectomy. Rubbery granulation tissue (white arrow) was noted in right mastoid antrum.

markedly. Although it would rarely occurr, its mortality rate is high enough to consider the possibility of intracranial complications in the treatment of otitis media.

Symptoms of otogenic brain abscess are generally mild in the early stage of initial encephalitis. Non-specific symptoms such as headache, nausea, vomiting, and fever can develop in the early stage of brain abscess. Headache is the most common symptom of otogenic brain abscess. Alteration of mental status and seizure can develop as a result of meningeal irritation and increased intracranial pressure. In rare cases, severe deterioration of motor function can be identified. ${ }^{7,8)}$

Otogenic brain abscess can be diagnosed with radiologic tests (CT and MRI). Mafee, et al. ${ }^{9)}$ highlight the important role of radiologic modalities in diagnosing patients who were clinically suspected of having otogenic brain abscess. In addition, serial review of imaging studies could be important to diagnosis. If symptoms and signs are not changed despite prolonged use of medication, serial radiologic study could be essential tests for the diagnosis of otogenic brain abscess or other intracranial complications. In this case, the abscess did not identify on the initial CT scan. With a high suspicion of intracranial complications, we did not hesitate to perform an additional image tests in order to reach the final diagnosis.

Streptococcus pneumonia, which is usually sensitive to empirical antibiotics, is the most common bacteria isolated from otogenic brain abscess. ${ }^{10)}$ Other aerobic bacteria (Staphylococcus, Proteus species), and anaerobic bacteria (Peptococcus, Peptostreptococcus, Bacteroides species) can also cause otogenic brain abscess. ${ }^{3)}$

On the other hand, Hafidh, et al. ${ }^{10)}$ reported that anaerobes are the most common organisms isolated from brain abscess followed by Staphylococcus, Proteus mirabilis and Pseudomonas. 
They suggest that the lack of specialized techniques to obtain anaerobic cultures attribute to the difficulty to isolate anaerobes from brain abscess. About $30 \%$ of otogenic brain abscess patients were negative in culture study. ${ }^{11)}$ Because of low sensitivity of culture modalities, it is very difficult to select susceptible antibiotics for otogenic brain abscess. We should consider that empirical antibiotic therapy cannot prevent intracranial complications perfectly. Empirical antibiotic therapy without culture and sensitivity tests might delay the diagnosis and treatment of acute otitis media and its possible complications, because it could mask symptoms. ${ }^{11)}$ Clinicians must pay attention to interpret the result of culture correctly in the end. The result of culture as well as drug sensitivity are the most important considerations in the treatment of brain abscess. In this case Streptococcus pneumonia was isolated and susceptible antibiotics were administered immediately. Despite the use of proper antibiotics, brain abscess eventually developed.

Surgical treatment consists of two categories, neurosurgical drainage of brain abscess and otologic surgery of primary focus, otits media. There is disagreement about the timing and the method of surgical intervention. ${ }^{12,13)}$ The necessity of the early mastoidectomy (within 24 hours) was emphasized for the sake of lowering mortality. Timely and active surgical removal of the infection source in the middle ear might enhance the treatment effect of antibiotics. ${ }^{2)}$

Advantages of neurosurgical drainage of brain abscess prior to otologic surgery were suggested by Singh and Maharaj. ${ }^{12)}$ They emphasized the importance of urgent neurosurgical drainage because if the mental state becomes worse, mortality of otogenic brain abscess will increase. Another study reported that if surgical procedures such as drainage of the brain abscess is done earlier, prognosis of the patient will improve. ${ }^{13)}$ On the contrary, the advantages of immediate otologic surgery with evacuation of the abscess through the mastoid cavity were suggested. ${ }^{3)}$ Single stage, transmastoid drainage of intracranial abscess showed the low morbidity and mortality rate combined with a shorter hospital stay. ${ }^{14)}$

Samuel, et al. ${ }^{4}$ suggested that there is no difference between therapeutic outcomes of otologic and neurosurgical operations performed together or done independantly. Moreover, non-operative treatment might be successfully applied in patients with a deteriorated general condition or deeply located abscess. ${ }^{15)}$ Various treatment strategies could be applied for the treatment of otogenic intracranial abscess according to the patient's condition and the characteristics of abscess.

In this case, serial brain imaging tests helped to detect the otologic brain abscess which could not be seen on the initial CT scans of initial presentation. Neurosurgical drainage was not considered in this patient, because the brain abscess was located in right thalamic area, deep portion of brain. Simple mastoidectomy was sufficient to eradicate the primary source of the infection. After mastoidectomy, the brain abscess was markedly decreased as seen in serial brain MRI scans. Despite the stuporous mental status at the time of presentation, the patient recovered properly with susceptible antibiotics and otologic surgery.

Following, it cannot be emphasized enough that serial image tests can be helpful to diagnose an otologic brain abscess with unusual clinical presentation. And we found that neurosurgical drainage of the brain abscess may not be neccessary if proper antibiotics are administered combined with otologic surgery, even in a deeply located brain abscess.

\section{REFERENCES}

1) Bradley PJ, Manning KP, Shaw MD. Brain abscess secondary to otitis media. J Laryngol Otol 1984;98:1185-91.

2) Szyfter W, Kruk-Zagajewska A, Borucki L, Bartochowska A. Evolution in management of otogenic brain abscess. Otol Neurotol 2012; 33:393-5.

3) Barry B, Delattre J, Vié F, Bedos JP, Géhanno P. Otogenic intracranial infections in adults. Laryngoscope 1999;109:483-7.

4) Samuel J, Fernandes CM, Steinberg JL. Intracranial otogenic complications: a persisting problem. Laryngoscope 1986;96:272-8.

5) Murthy PS, Sukumar R, Hazarika P, Rao AD, Mukulchand, Raja A. Otogenic brain abscess in childhood. Int J Pediatr Otorhinolaryngol 1991;22:9-17.

6) Kulai A, Ozatik N, Topçu I. Otogenic intracranial abscesses. Acta Neurochir (Wien) 1990;107:140-6.

7) Chung JW, Lee SW, Song HM, Kim JH. Otogenic brain abscess: recent experience of 13 cases. Korean J Otolaryngol-Head Neck Surg 2004;47:1054-9.

8) Ahn JK, Hwang CH, Bae WY, Lee HJ, Kang MK, Kim LS. Clinical presentations and managements of otogenic brain abscess. J Clinical Otolaryngol 2005;16:240-6.

9) Mafee MF, Valvassori GE, Kumar A, Levin BC, Siedentop KH, Raju S. Otogenic intracranial inflammations: role of CT. Otolaryngol Clin North Am 1988;21:245-63.

10) Hafidh MA, Keogh I, Walsh RM, Walsh M, Rawluk D. Otogenic intracranial complications. A 7-year retrospective review. Am J Otolaryngol 2006;27:390-5.

11) Dhooge IJ, Albers FW, Van Cauwenberge PB. Intratemporal and intracranial complications of acute suppurative otitis media in children: renewed interest. Int J Pediatr Otorhinolaryngol 1999;49 Suppl 1:S109-14.

12) Singh B, Maharaj TJ. Radical mastoidectomy: its place in otitic intracranial complications. J Laryngol Otol 1993;107:1113-8.

13) Kurien M, Job A, Mathew J, Chandy M. Otogenic intracranial abscess: concurrent craniotomy and mastoidectomy--changing trends in a developing country. Arch Otolaryngol Head Neck Surg 1998; 124:1353-6.

14) Morwani KP, Jayashankar N. Single stage, transmastoid approach for otogenic intracranial abscess. J Laryngol Otol 2009;123:1216-20.

15) Rosenblum ML, Hoff JT, Norman D, Edwards MS, Berg BO. Nonoperative treatment of brain abscesses in selected high-risk patients. J Neurosurg 1980;52:217-25. 